\title{
Serum Albumin Levels are a Predictor of COVID-19 Patient Prognosis: Evidence from a Single Cohort in Chongqing, China
}

\author{
Yuan $X u^{1-4}$ \\ Huan Yang ${ }^{2,5}$ \\ Jing Wang ${ }^{6}$ \\ Xiaoying $\mathrm{Li}$ iD ${ }^{2,5}$ \\ Chengjun $X{ }^{6}$ \\ Changchun $\mathrm{Niu}^{2}$ \\ Pu Liao ${ }^{1,2}$ \\ 'Chongqing Medical University, \\ Chongqing, 4000 I4, People's Republic of \\ China; ${ }^{2}$ Department of Clinical \\ Laboratory, Chongqing General Hospital, \\ University of Chinese Academy of \\ Sciences, Chongqing, 4000I4, People's \\ Republic of China; ${ }^{3}$ Chongqing College, \\ University of Chinese Academy of \\ Sciences, Chongqing, 4000I4, People's \\ Republic of China; ${ }^{4}$ Department of \\ Clinical Laboratory, Affiliated Hospital of \\ North Sichuan Medical College, \\ Nanchong, 637000, People's Republic of \\ China; ${ }^{5}$ Southwest Medical University, \\ Luzhou, 646000, People's Republic of \\ China; ${ }^{6}$ Department of Clinical \\ Laboratory, Chongqing Public Health \\ Medical Center, Chongqing, 400036, \\ People's Republic of China
}

Background: COVID-19 infections are still at pandemic levels globally and there are currently no specific drugs to treat these infections. Previous studies have demonstrated that serum albumin levels were abnormally low in COVID-19 patients and might be used as a prognosis biomarker. Supplemental albumin has been used as an experimental therapeutic method. However, dynamic evaluation of albumin in patients with COVID19 was limited and whether serum albumin could predict the prognosis of these patients is unknown.

Methods: We enrolled 79 COVID-19 patients in the present study and reviewed electronic medical laboratory records. Data was processed using SPSS software (Version 20.0) and correlation analysis was performed between serum albumin and other clinical and laboratory findings.

Results: Serum albumin levels were gradually decreased both in severe and non-severe COVID-19 patients. Moreover, $17.7 \%$ of the patients presented with hypoalbuminemia at least one time during 3 consecutive weekly time points. The hypoalbuminemia group displayed more severe disease and comorbidity that included fever, fatigue, headache, and dizziness on admission. Moreover, serum albumin levels were positively correlated with lymphocyte and $\mathrm{RBC}$ numbers, $\mathrm{Hb}$ and prealbumin levels as well as with total $\mathrm{T}$ cell numbers and the presence of $\mathrm{CD}^{+}$and $\mathrm{CD}^{+} \mathrm{T}$ cells. In contrast, there was a negative correlation with C-reactive protein levels and this was an indicator of patient recovery.

Conclusion: Our results demonstrated that hypoalbuminemia was common in COVID-19 patients and its levels were linked to disease severity. Patients with fever, fatigue and headache or dizziness on admission were more likely to experience hypoalbuminemia. Dynamic monitoring of serum albumin is therefore necessary and should be performed during COVID-19 patient treatments as a tool for evaluating the prognosis of COVID-19 infections.

Keywords: COVID-19, SARS-CoV-2, albumin, liver function, prognosis

\section{Introduction}

The recent outbreak of COVID-19 (SARS-CoV-2) has resulted in more than 100 million infections and 3.2 million deaths. Infection by this virus mimics the clinical phenotypes of SARS in its severity that includes pneumonia and high mortality. ${ }^{1,2}$ Additionally, the lungs are not unique targets of SARS-CoV -2 and other targets include the heart, liver, kidney and the male reproductive 
system. ${ }^{3-7}$ These studies have suggested that COVID19 might cause systemic disease.

A large cohort analysis with 2623 patients demonstrated a marked hypoalbuminemia that occurred upon admission in patients that were non-critically ill (38.2\%), critically ill (71.2\%) and that had succumbed to the illness $(82.4 \%)$. These numbers rose to 45.9 , 77.7 , and $95.6 \%$ during hospitalization, respectively. ${ }^{8}$ This study also indicated that hypoalbuminemia caused by COVID-19 infection was the result of a cytokine release syndrome or a "cytokine storm" that was associated with poor prognosis in these patients. Age and comorbidities were also associated with poor prognosis. ${ }^{9,10}$ In contrast, another study demonstrated that hypoalbuminemia was associated with COVID-19 infection outcomes that was age and comorbidity independent. ${ }^{11}$ Low albumin levels were also associated with poor prognosis of COVID-19 patients as measured by length of hospitalization and mortality. ${ }^{12}$ Importantly, the link between hypoalbuminemia and infection severity has not been followed-up and it is not known whether serum albumin is a useful biomarker to monitor infection severity. In this study, we retrospectively assessed serum albumin levels that were collected over time in patients diagnosed with COVID-19 disease to analyze the role of hypoalbuminemia in disease progression and prognosis.

\section{Methods}

\section{Subjects}

This study was a retrospective single-center analysis including patients suffered from COVID-19 disease hospitalized in Chongqing Public Health Medical Center from January 2020 to May 2020. Diagnosis of COVID-19 and clinical classification was made according to the new coronavirus pneumonia diagnosis and treatment plan (trial 5th version) developed by the National Health Committee of the People's Republic of China (http://www.nhc.gov.cn/). Based on these guidelines, patients were defined as suspected COVID-19 when they met the following clinical criteria: (1) Mild: mild symptoms without pneumonia; (2) Typical: fever or respiratory tract symptoms with pneumonia; (3) Severe: fulfill any of the three criteria: respiratory distress, respiratory rate R 30 times/min; means oxygen saturation $93 \%$ in resting state; arterial blood oxygen partial pressure/oxygen concentration $300 \% \mathrm{~mm} \mathrm{Hg}(1 \mathrm{mmHg}=0.133 \mathrm{kPa})$; (4) Critical: fulfill any of the three criteria: respiratory failure and require mechanical ventilation; shock incidence; admission to ICU with other organ failure. The SARS-CoV-2 virus nucleic acid detection in throat swab was positive for all patients in this study. In summary, we enrolled 79 patients that were divided into 17 severe and 62 of non-severe cases. This study was approved by the ethics committee of Chongqing People's Hospital (S2020-021-01).

\section{Data Collection}

Electronic medical records were used as the data source and included epidemiological history, demographic characteristics, comorbidities and clinical symptoms. Laboratory tests included routine peripheral blood and urine screening, coagulation profiles and serum biochemical and immunological analyses. Additionally, therapeutic measures including use of antibiotics, oxygen therapy, fluid and nutritional support and application of glucocorticoids were also included in the present study. The serum albumin levels $<35 \mathrm{~g} / \mathrm{L}$ was defined as hypoalbuminemia. All laboratory findings were collected from COVID-19 patients at three time points including weeks 1,2 and 3-4 from onset or the first positive nucleic acid test to blood collection. To ensure the accuracy and completeness of the data, all authors reviewed the extracted data and revised errors immediately by checking the original case data after the errors were found.

\section{Statistical Analysis}

Continuous variables were described as mean $\pm \mathrm{SD}$ or median [IQR]. The Student's $t$ test and Mann-Whitney $U$-test were used to analyze continuous variables based on normal distributed data or non-normal distributed data, respectively. Categorical variables were presented as frequency rates and percentages and analyzed using the $\chi^{2}$ test or Fisher's exact test as appropriate. All of the data was performed in SPSS 22.0 software (IBM, Chicago, Ill, USA) and a two-sided $p$ value of 0.05 or less was considered statistically significant. 


\section{Results}

\section{The Clinical Characteristics of Patients}

Our group of 79 patients included 38 male and 41 female with a median age of 45 (range 4 to 80 ). Nonsevere cases included $17(21.5 \%)$ patients and 62 $(78.5 \%)$ were defined as severe cases. All of the patients were further divided into a normal albumin group and hypoalbuminemia group to evaluate the association of albumin with disease severities. We identified 65 normal and 14 patients with hypoalbuminemia and in the latter, albumin levels were significantly less in the severe group (71.4\%) than in the nonsevere group (10.8\%). This revealed that hypoalbuminemia was more common in COVID-19 patients with severe disease (Table 1).

Patients that required oxygen inhalation therapies were also more common in the hypoalbuminemia group and these patents also experienced higher incidences of fever $(13,92.9 \%)$, fatigue $(8,57.1 \%)$, and chest tightness $(8,57.1 \%)$. Overall, the most common comorbidities found in the infected patients were hypertension, diabetes, hematological, digestive and respiratory system diseases. These comorbidities were not significantly between the normal albumin and hypoalbuminemia groups. Cause of the limited case of each subgroup, the results should be further validated in other cohorts. Furthermore, the most significant complications for the hypoalbuminemia group were respiratory and hematological system involvement and skin disease. All 79 patients received antiviral treatment and more patients received corticosteroids, an immunomodulator and antibiotic treatment in the hypoalbuminemia (Table 1).

\section{Association of Serum Albumin with Hematological Parameters}

During 3-4 weeks of hospitalization and treatment, serum albumin levels for all patients had risen compared with week 2. However, severe COVID-19 infections resulted in lower serum albumin levels overall for the severe patients at the 3 time points (Figure 1). Hemoglobin levels were lower in the hypoalbuminemia group within the 3 time points while $C$ reactive protein (CRP) levels were higher compared with the normal controls (Table 2). Lymphocyte counts for the hypoalbuminemia group were elevated by week 2 and gradually decreased by weeks 3 and 4 (Figure 2A). Moreover, erythrocyte counts and hemoglobin levels for the hypoalbuminemia group were significantly different within the first week and dropped more rapidly as time progressed compared with the normal albumin group (Figure 2C and E). CRP was elevated in most of the severe COVID-19 patients while the normal albumin group maintained low CRP levels throughout the course of study. The high CRP levels in the hypoalbuminemia group declined over weeks 2 4 (Figure 2G). Furthermore, serum albumin in COVID19 patients was positively correlated with lymphocyte count, $\mathrm{Hb}$ levels and $\mathrm{RBC}$ counts and negatively correlated with CRP levels (Figure 2B, D, F, H). These results indicated that serum albumin might be a predicator of hematological parameters for COVID19 patients.

\section{Association of Serum Albumin with Biomarkers from Serum Biochemical Tests}

Our group of patients with hypoalbuminemia also displayed prealbumin levels that were significantly lower than the normal albumin controls over the whole study period. In addition, AST but not ALT levels were higher in patients with hypoalbuminemia and these levels were significantly reduced within 3-4 weeks of treatment. Kidney functional parameters including urea nitrogen, uric acid, creatinine, $\beta 2$-microglobulin and cystatin $\mathrm{C}$ were not markedly different over time between the normal albumin and hypoalbuminemia groups (Table 3). These results indicated that liver function, especially those that were involved in protein synthesis functions were damaged in patients with hypoalbuminemia. A dynamic analysis of these parameters also indicated that in both our test groups, prealbumin levels gradually increased over hospitalization time but recovery for patients with hypoalbuminemia was significantly slower than the normal albumin group (Figure 3A). This indicated that liver functional recovery was still weak in the hypoalbuminemia group. Moreover, AST levels were recovered in the latter group over time while ALT levels showed no significant change (Figure $3 \mathrm{C}$ and E). Furthermore, 
Table I Clinical Features of Patients with COVID-I9

\begin{tabular}{|c|c|c|c|c|}
\hline \multirow[t]{2}{*}{ Indicators } & \multicolumn{3}{|c|}{ Albumin } & \multirow[t]{2}{*}{ p value } \\
\hline & $\begin{array}{c}\text { Total }(n=79 \text {, } \\
39.2 \pm 4.1)\end{array}$ & $\begin{array}{c}\text { Normal }(n=65, \\
40.7 \pm 2.9)\end{array}$ & $\begin{array}{c}\text { Hypoalbuminemia } \\
(n=\mid 4,32.6 \pm 1.5)\end{array}$ & \\
\hline \multicolumn{5}{|l|}{ Gender } \\
\hline Male & $38(48.1 \%)$ & $30(46.2 \%)$ & $8(57.1 \%)$ & 0.455 \\
\hline Female & $4 I(5 I .9 \%)$ & $35(53.8 \%)$ & $6(42.9 \%)$ & \\
\hline \multicolumn{5}{|l|}{ Severity } \\
\hline Non-severe & $62(78.5 \%)$ & $58(89.2 \%)$ & $4(28.6 \%)$ & 0.000 \\
\hline Severe & $\mid 7(2 \mid .5 \%)$ & $7(10.8 \%)$ & $10(71.4 \%)$ & \\
\hline Cigarette smoking & $17(2 \mid .5 \%)$ & $14(2 \mid .5 \%)$ & $3(21.4 \%)$ & 1.000 \\
\hline Alcohol consumption & $2 \mathrm{I}(26.6 \%)$ & $17(8 \mid .0 \%)$ & $4(19.0 \%)$ & 1.000 \\
\hline Oxygen inhalation & $44(55.7 \%)$ & $31(47.7 \%)$ & $13(92.9 \%)$ & 0.002 \\
\hline \multicolumn{5}{|c|}{ Days from Onset/first nucleic acid positive to Admission } \\
\hline M $\pm S . D$. & $3.5 \pm 2.1$ & $3.4 \pm 2.1$ & $3.8 \pm 1.8$ & 0.440 \\
\hline Median (IQR) & $3.0(2.0-5.0)$ & $3.0(2.0-5.0)$ & $4.0(2.3-5.8)$ & \\
\hline Range & 0-9.0 & $0-9.0$ & $1.0-6.0$ & \\
\hline \multicolumn{5}{|c|}{$\begin{array}{l}\text { Days from Onset/first nucleic acid positive to First } \\
\text { consecutive nucleic acid negative }\end{array}$} \\
\hline $\mathrm{M} \pm \mathrm{SD}$ & $20.9 \pm 9.9$ & $20.9 \pm 10.3$ & $20.9 \pm 8.1$ & 0.919 \\
\hline Median (IQR) & $20.0(14.0-27.0)$ & $20(14.0-28.0)$ & $19.0(16.5-23.0)$ & \\
\hline Range & $2.0-44.0$ & $2.0-44.0$ & $10.0-44.0$ & \\
\hline \multicolumn{5}{|l|}{ Days from Admission to Discharge } \\
\hline $\mathrm{M} \pm \mathrm{SD}$ & $22.4 \pm 8.9$ & $21.4 \pm 8.7$ & $26.7 \pm 8.2$ & 0.056 \\
\hline Median (IQR) & $21.0(14.0-29.0)$ & $20(13.0-28.0)$ & $26.0(20.5-33.8)$ & \\
\hline Range & $6.0-42.0$ & $6.0-42.0$ & $13.0-42.0$ & \\
\hline \multicolumn{5}{|c|}{ Days from Onset/first nucleic acid positive to Discharge } \\
\hline $\mathrm{M} \pm \mathrm{SD}$ & $25.9 \pm 9.1$ & $24.9 \pm 8.9$ & $30.5 \pm 8.4$ & 0.073 \\
\hline Median (IQR) & $25.0(17.5-32.0)$ & $23.0(\mid 7.0-31.0)$ & $31.0(23.5-36.8)$ & \\
\hline Range & $7.0-47.0$ & $7.0-46.0$ & $14.0-47.0$ & \\
\hline \multicolumn{5}{|l|}{ Symptoms } \\
\hline Fever & $47(59.5 \%)$ & $34(52.3 \%)$ & $13(92.9 \%)$ & 0.005 \\
\hline Cough & $4 I(51.9 \%)$ & $32(49.2 \%)$ & $9(64.3 \%)$ & 0.306 \\
\hline Sputum & $25(31.6 \%)$ & $21(32.3 \%)$ & $4(28.6 \%)$ & 1.000 \\
\hline Pharyngalgia & $12(15.2 \%)$ & II (I6.9\%) & $(7.1 \%)$ & 0.607 \\
\hline Headache or dizzy giddy & $16(19.0 \%)$ & $10(15.4 \%)$ & $6(42.9 \%)$ & 0.051 \\
\hline Fatigue & $21(26.6 \%)$ & $13(20.0 \%)$ & $8(57.1 \%)$ & 0.012 \\
\hline Diarrhea & $7(8.9 \%)$ & $5(7.7 \%)$ & $2(14.3 \%)$ & 0.431 \\
\hline Chest tightness & $22(27.8 \%)$ & $14(2 \mid .5 \%)$ & $8(57.1 \%)$ & 0.018 \\
\hline \multicolumn{5}{|l|}{ Comorbidity } \\
\hline Hypertension & $9(11.4 \%)$ & $6(9.2 \%)$ & $3(21.4 \%)$ & $0.40 \mathrm{I}$ \\
\hline Diabetes & $5(6.3 \%)$ & $3(4.6 \%)$ & $2(14.3 \%)$ & 0.458 \\
\hline Hematological System diseases & $4(5.1 \%)$ & $2(3.1 \%)$ & $2(14.3 \%)$ & 0.288 \\
\hline Digestive system diseases & $7(8.9 \%)$ & $5(7.7 \%)$ & $2(14.3 \%)$ & 0.788 \\
\hline Other Cardiovascular systemdiseases & $2(2.5 \%)$ & $\mathrm{I}(\mathrm{I} .5 \%)$ & $\mathrm{I}(7.1 \%)$ & 0.785 \\
\hline Respiratory system diseases & $\mathrm{I}(\mathrm{I} .3 \%)$ & $\mathrm{I}(\mathrm{I} .5 \%)$ & $0(0.0 \%)$ & 1.000 \\
\hline Other Metabolicdiseases & $3(3.8 \%)$ & $2(3.1 \%)$ & $\mathrm{I}(7.1 \%)$ & 1.000 \\
\hline
\end{tabular}

(Continued) 
Table I (Continued).

\begin{tabular}{|c|c|c|c|c|}
\hline \multirow[t]{2}{*}{ Indicators } & \multicolumn{3}{|c|}{ Albumin } & \multirow[t]{2}{*}{ p value } \\
\hline & $\begin{array}{c}\text { Total }(n=79, \\
39.2 \pm 4.1)\end{array}$ & $\begin{array}{c}\text { Normal }(n=65, \\
40.7 \pm 2.9)\end{array}$ & $\begin{array}{c}\text { Hypoalbuminemia } \\
(n=14,32.6 \pm 1.5)\end{array}$ & \\
\hline \multicolumn{5}{|l|}{ Complications } \\
\hline Respiratory system & $21(26.6 \%)$ & $12(18.5 \%)$ & $9(64.3 \%)$ & 0.001 \\
\hline Digestive system & II(I3.9\%) & $7(10.8 \%)$ & $4(28.6 \%)$ & 0.187 \\
\hline Metabolicdiseases & $3(3.8 \%)$ & $3(4.6 \%)$ & $0(0.0 \%)$ & 0.961 \\
\hline Hematological System & $4(5.1 \%)$ & $\mathrm{I}(\mathrm{I} .5 \%)$ & $3(21.4 \%)$ & 0.016 \\
\hline Immune system & $4(5.1 \%)$ & $2(3.1 \%)$ & $2(14.3 \%)$ & 0.288 \\
\hline Urinary system & $\mathrm{I}(\mathrm{I} .3 \%)$ & $0(0.0 \%)$ & $\mathrm{I}(7.1 \%)$ & 0.395 \\
\hline Neuropsychological system & $\mathrm{I}(\mathrm{I} .3 \%)$ & $\mathrm{I}(\mathrm{I} .5 \%)$ & $0(0.0 \%)$ & 1.000 \\
\hline Skin diseases & $3(3.8 \%)$ & $0(0.0 \%)$ & $3(21.4 \%)$ & 0.002 \\
\hline Cardiovascular system & $3(3.8 \%)$ & $\mathrm{I}(\mathrm{I} .5 \%)$ & $2(14.3 \%)$ & 0.136 \\
\hline \multicolumn{5}{|l|}{ Chest CT-no (\%) } \\
\hline Involvement of chest radiographs & $58(73.4 \%)$ & $45(69.2 \%)$ & $13(92.9 \%)$ & 0.138 \\
\hline \multicolumn{5}{|l|}{ Treatment } \\
\hline Antiviral & $78(98.7 \%)$ & $64(98.5 \%)$ & $14(100 \%)$ & 1.000 \\
\hline Traditional Chinese medicine & $67(84.8 \%)$ & $54(83.1 \%)$ & $13(92.9 \%)$ & 0.607 \\
\hline Corticosteroid & $13(16.5 \%)$ & $4(6.2 \%)$ & $9(64.3 \%)$ & 0.000 \\
\hline Immunomodulator & $17(2 \mid .5 \%)$ & $8(12.3 \%)$ & $9(64.3 \%)$ & 0.000 \\
\hline Antibiotic & $18(22.8 \%)$ & $9(13.8 \%)$ & $9(64.3 \%)$ & 0.000 \\
\hline
\end{tabular}

prealbumin was positively correlated and AST was demonstrated that serum albumin could be used as negatively correlated with albumin concentration a biomarker to evaluate liver damage in COVID-19 while ALT was not (Figure 3B, D, F). These results patients.

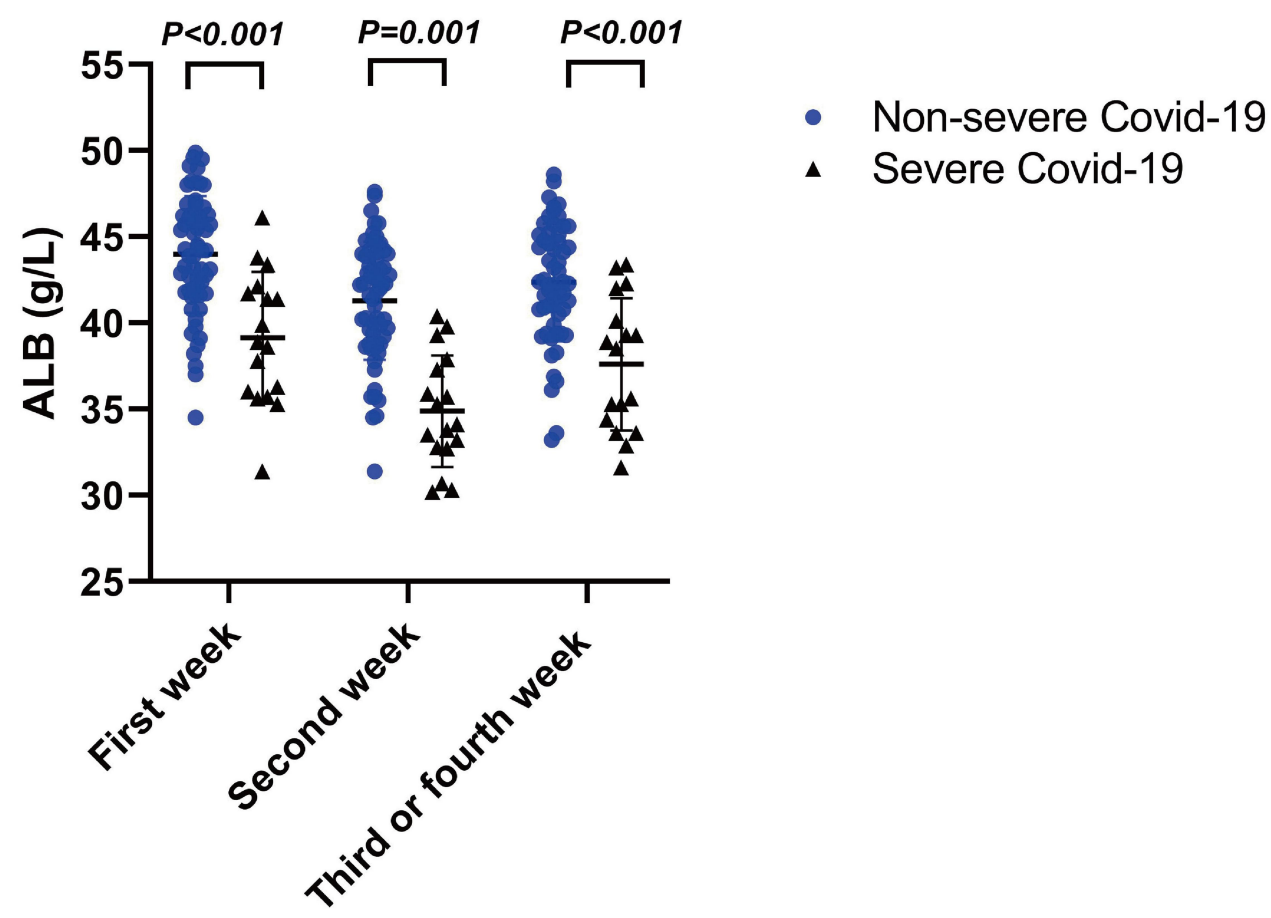

Figure I Serum albumin levels between severe and non-severe patients at 3 time-points. Statistically significant at $P$ value less than 0.05 . 


\begin{tabular}{|c|c|c|c|c|c|c|c|c|c|c|}
\hline \multicolumn{2}{|l|}{$\frac{\mathscr{g}}{\frac{\partial}{N}}$} & 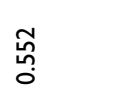 & $\frac{0}{\pi}$ & $\bar{o}$ & o̊ & ڤ్ণ & : & চ̊. & 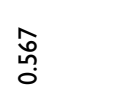 & চ̊ \\
\hline \multirow{2}{*}{ 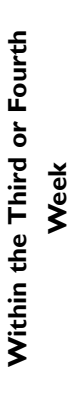 } & 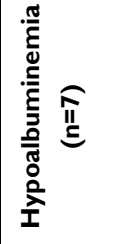 & 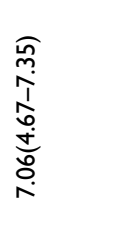 & 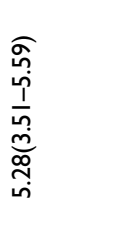 & 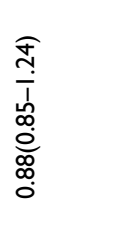 & 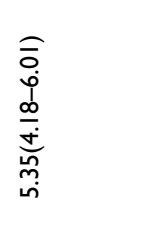 & $\begin{array}{l}\widehat{\sigma} \\
0 \\
0 \\
1 \\
0 \\
0 \\
0 \\
0 \\
0 \\
0 \\
0\end{array}$ & 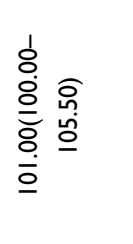 & 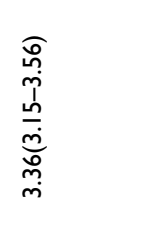 & 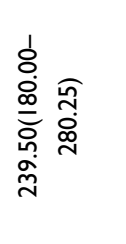 & 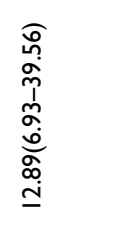 \\
\hline & 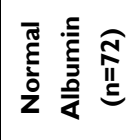 & 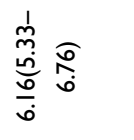 & 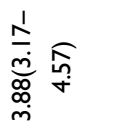 & 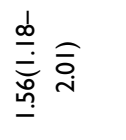 & 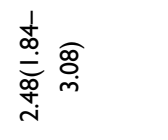 & 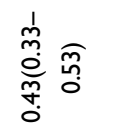 & 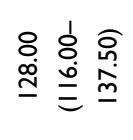 & 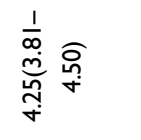 & 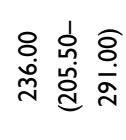 & 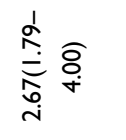 \\
\hline ڤّ̆ & $\underset{11}{0}$ & 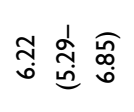 & 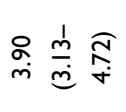 & 菅舍 & 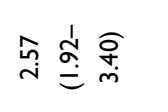 & 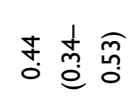 & 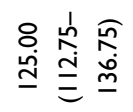 & 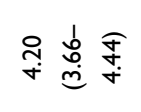 & 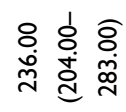 & 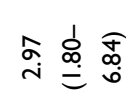 \\
\hline \multicolumn{2}{|l|}{$\begin{array}{l}\frac{0}{2} \\
\frac{0}{2} \\
0\end{array}$} & $\frac{\infty}{0}$ & ঃ & ঃ & ঃ & 总 & ڤ̊ & $\bar{o}$ & 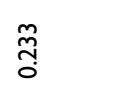 & : \\
\hline \multirow{2}{*}{ 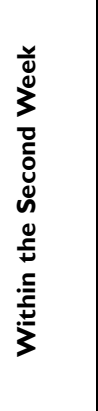 } & 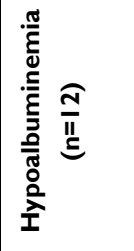 & 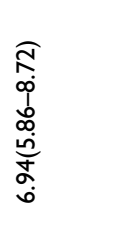 & 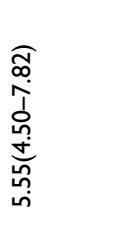 & $\begin{array}{l}\widehat{0} \\
\hat{0} \\
0 \\
0 \\
0 \\
0 \\
0 \\
\stackrel{0}{0} \\
0\end{array}$ & 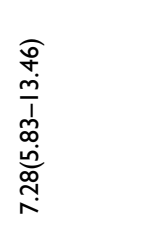 & 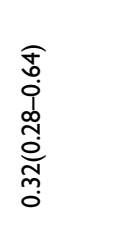 & 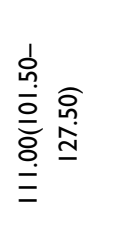 & 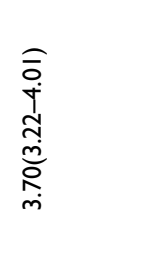 & 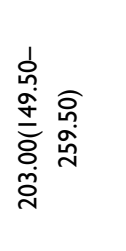 & 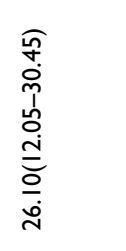 \\
\hline & 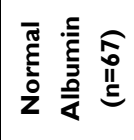 & 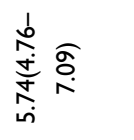 & 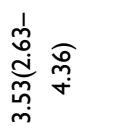 & 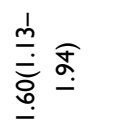 & 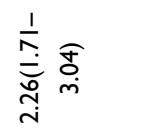 & 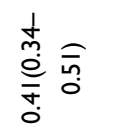 & 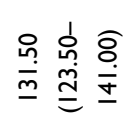 & 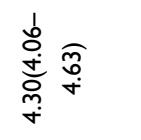 & 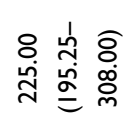 & 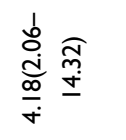 \\
\hline હֶّ & 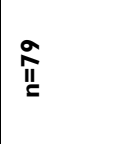 & 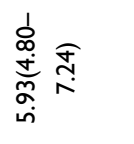 & 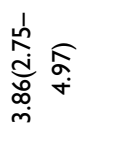 & 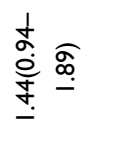 & 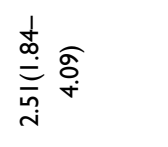 & 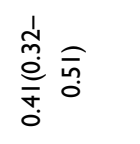 & 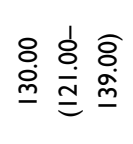 & 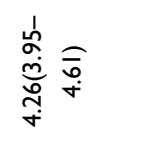 & 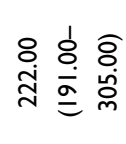 & 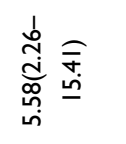 \\
\hline \multicolumn{2}{|l|}{$\begin{array}{l}\frac{0}{\frac{D}{N}} \\
\frac{2}{2} \\
0\end{array}$} & 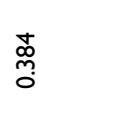 & 号 & $\begin{array}{l}\infty \\
\stackrel{\infty}{0} \\
0\end{array}$ & $\frac{1}{a}$ & $\frac{d}{8}$ & $\begin{array}{l}\text { İ } \\
\text { Oे }\end{array}$ & î̀. & 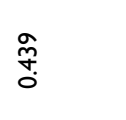 & ¿ั. \\
\hline \multirow{2}{*}{ 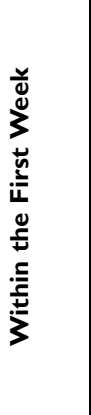 } & 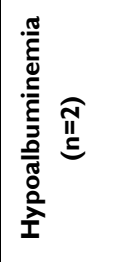 & 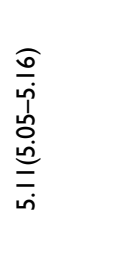 & 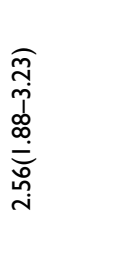 & 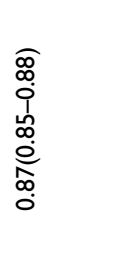 & 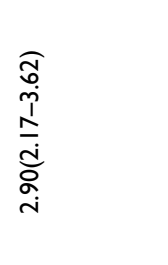 & 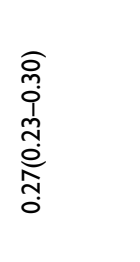 & 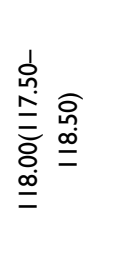 & 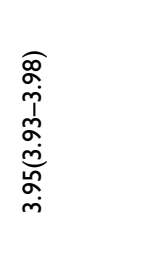 & 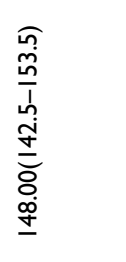 & 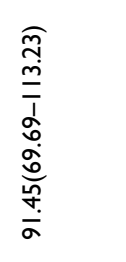 \\
\hline & 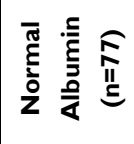 & 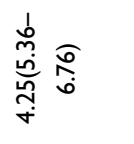 & 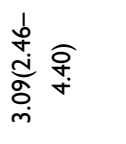 & 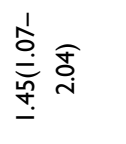 & 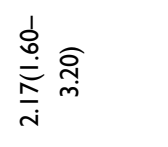 & 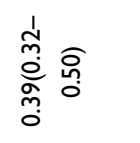 & 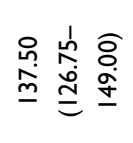 & 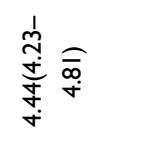 & 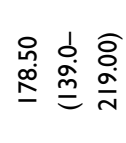 & 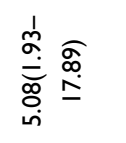 \\
\hline 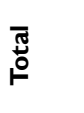 & î̀ & 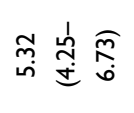 & ồ & $\stackrel{p}{=} \stackrel{+}{=} \widehat{\bar{\sigma}}$ & 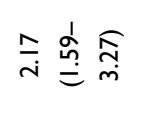 & $\stackrel{\infty}{0} \stackrel{\frac{1}{m}}{\stackrel{m}{0}} \stackrel{\hat{h}}{0}$ & 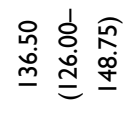 & 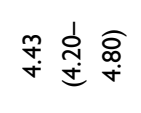 & 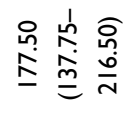 & $\bar{m} \stackrel{\infty}{\stackrel{\infty}{=}} \frac{\widehat{\infty}}{\bar{N}}$ \\
\hline \multicolumn{2}{|l|}{ 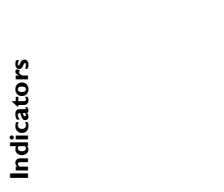 } & 曼 & 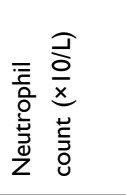 & 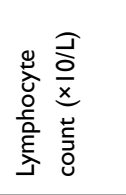 & 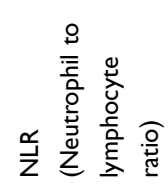 & 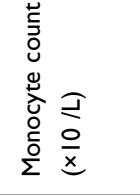 & 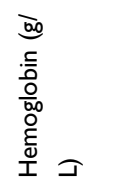 & 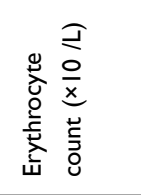 & 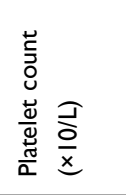 & 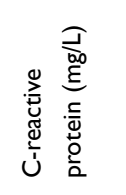 \\
\hline
\end{tabular}


A

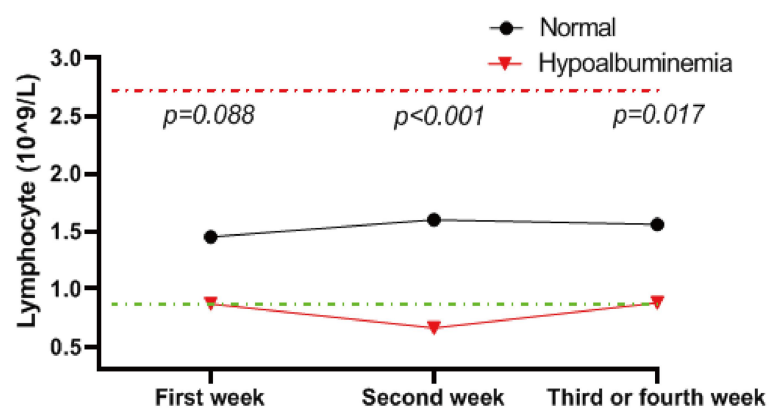

C

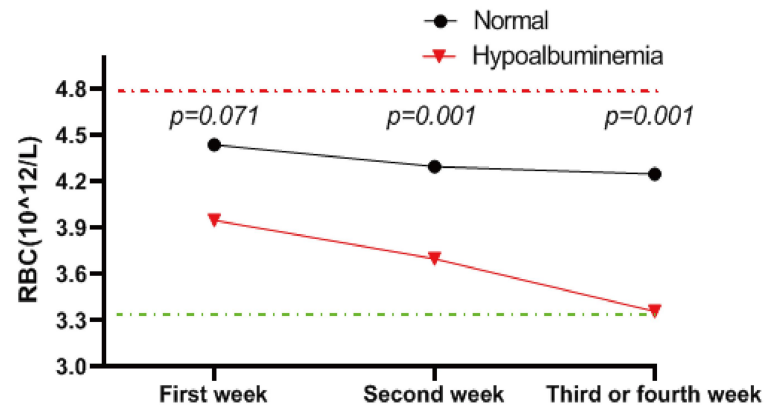

E

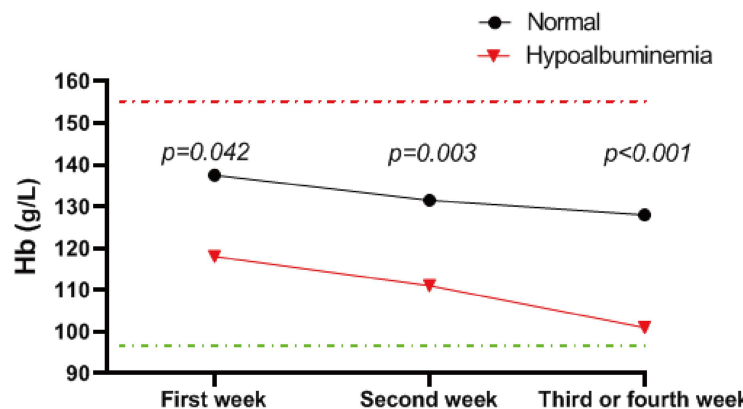

G

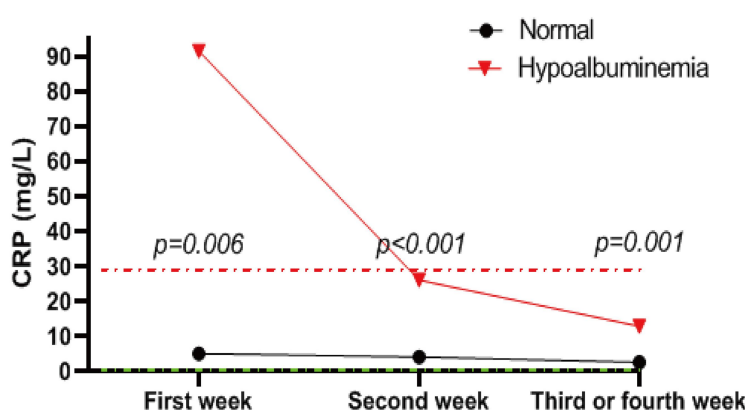

B

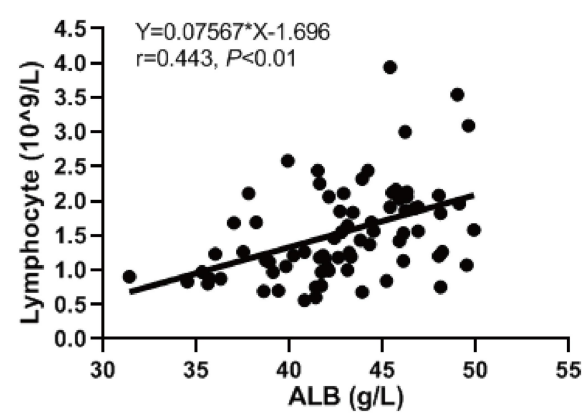

D

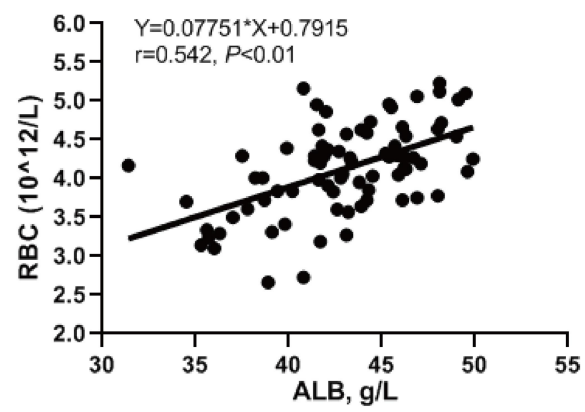

F

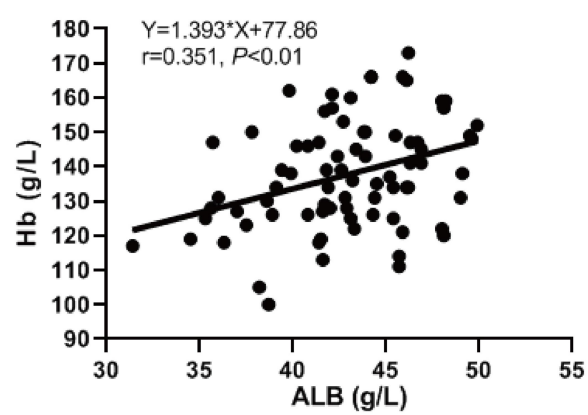

H

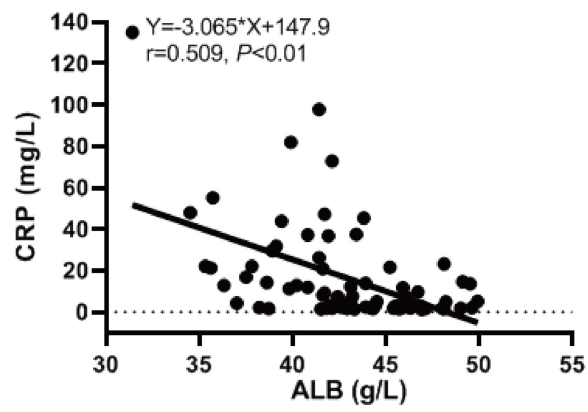

Figure 2 Dynamic analysis of peripheral blood parameters for COVID-19 patients. (A, C, E, G) Levels of Lymphocytes count (A), RBC count (C), Hb concentration (E), and CRP concentration (G) in COVID patients with albumin reduction and normal albumin at three time points, including acute phase, advanced phase, and recovery phase. (B, D, F, H) The correlation of serum albumin with Lymphocytes count (B), RBC (D), Hb concentration (F), and CRP concentration (H) in COVID patients. Statistically significant at $P$ value less than 0.05 . 


\begin{tabular}{|c|c|c|c|c|c|c|c|c|c|}
\hline \multicolumn{2}{|l|}{$\begin{array}{l}\frac{0}{\frac{D}{N}} \\
\frac{1}{2} \\
a\end{array}$} & ০̊ & $\stackrel{\substack{m \\
0}}{0}$ & ָ̊ & $\begin{array}{l}\overline{0} \\
\text { o. }\end{array}$ & $\frac{}{ㅁ}$ & $\begin{array}{c}+ \\
\infty \\
\infty \\
0\end{array}$ & $\frac{\circ}{\circ}$ & $\overline{\bar{o}}$ \\
\hline \multirow{2}{*}{ 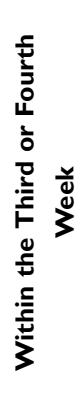 } & 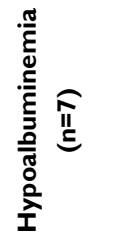 & 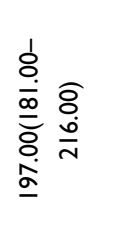 & 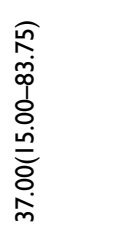 & 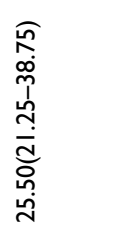 & $\begin{array}{l}\text { fo } \\
0 \\
0 \\
0 \\
0 \\
0 \\
0 \\
0 \\
0\end{array}$ & 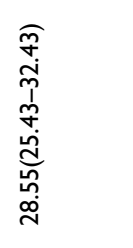 & 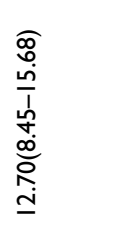 & 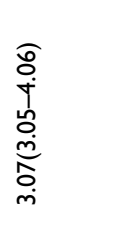 & 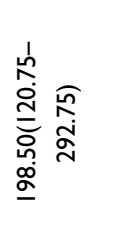 \\
\hline & 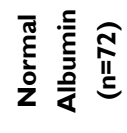 & 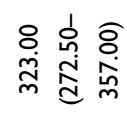 & 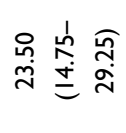 & 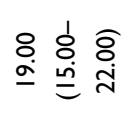 & 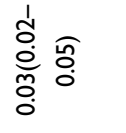 & 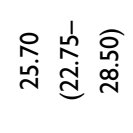 & 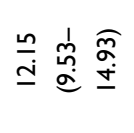 & 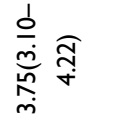 & 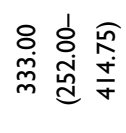 \\
\hline 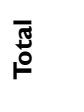 & $\underset{\text { II }}{\stackrel{0}{\varepsilon}}$ & 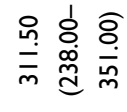 & 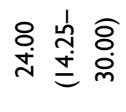 & 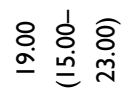 & 苂 宫 & 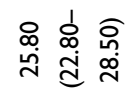 & 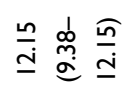 & 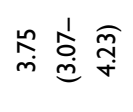 & 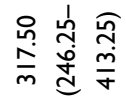 \\
\hline \multicolumn{2}{|l|}{$\begin{array}{l}\frac{0}{\frac{0}{N}} \\
\frac{1}{2} \\
0\end{array}$} & : & $\frac{\sigma}{\overline{0}}$ & $\overline{\underline{\Lambda}}$ & o̊ & $\stackrel{\text { on }}{\stackrel{0}{0}}$ & $\begin{array}{l}\bar{\sigma} \\
\text { Oे }\end{array}$ & $\stackrel{\substack{\tilde{O} \\
0}}{0}$ & $\overline{\bar{o}}$ \\
\hline \multirow{2}{*}{ 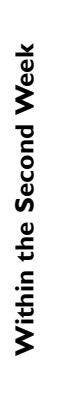 } & 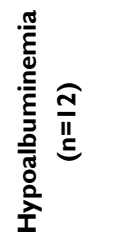 & 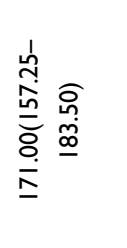 & 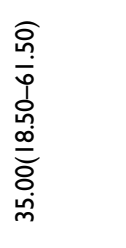 & 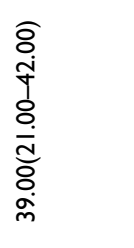 & 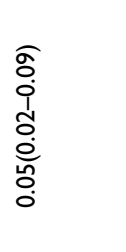 & 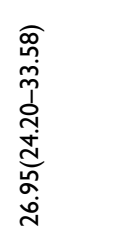 & 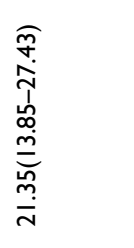 & 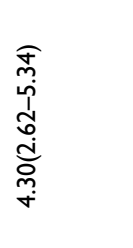 & 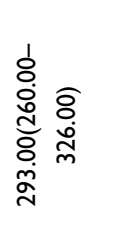 \\
\hline & 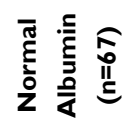 & 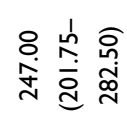 & 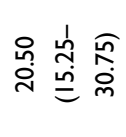 & 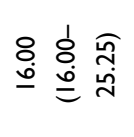 & 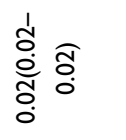 & 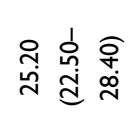 & 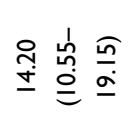 & 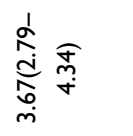 & 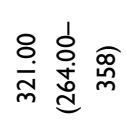 \\
\hline ङ્ّ & $\stackrel{0}{i=}$ & 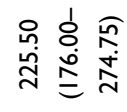 & 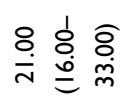 & 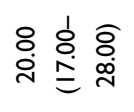 & 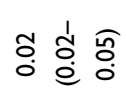 & 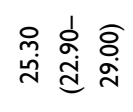 & 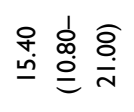 & 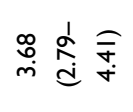 & 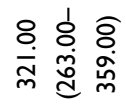 \\
\hline \multicolumn{2}{|l|}{$\begin{array}{l}\frac{0}{\frac{D}{N}} \\
\frac{1}{2} \\
0\end{array}$} & ठ̊. & ڤু & $\stackrel{+}{\stackrel{N}{o}}$ & $\stackrel{\text { o̊ }}{\circ}$ & $\frac{f}{0}$ & $\begin{array}{l}\hat{\mathrm{o}} \\
\stackrel{0}{0}\end{array}$ & $\frac{\infty}{\infty}$ & $\stackrel{\bar{\lambda}}{o}$ \\
\hline \multirow{2}{*}{ 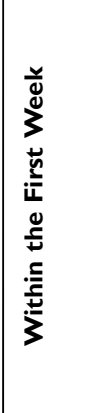 } & 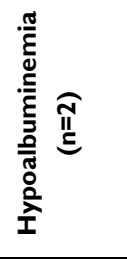 & 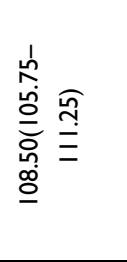 & 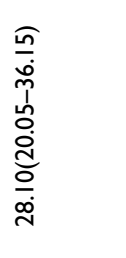 & 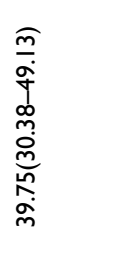 & $\begin{array}{l}\frac{\frac{\pi}{0}}{0} \\
\frac{1}{0} \\
\frac{d}{0}\end{array}$ & 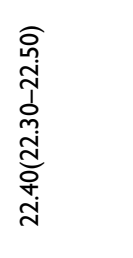 & 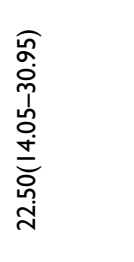 & 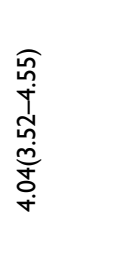 & 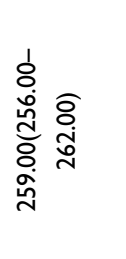 \\
\hline & 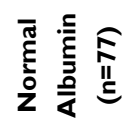 & 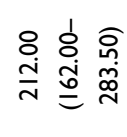 & 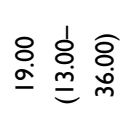 & 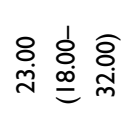 & 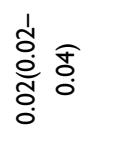 & 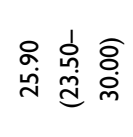 & 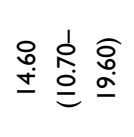 & 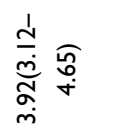 & 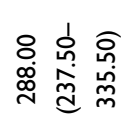 \\
\hline बֶّ & $\stackrel{a}{11}$ & 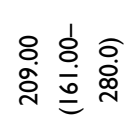 & 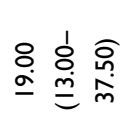 & 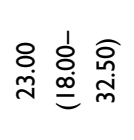 & 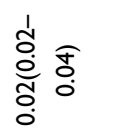 & 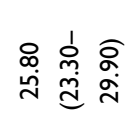 & 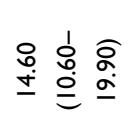 & 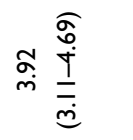 & 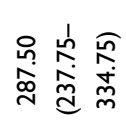 \\
\hline \multicolumn{2}{|l|}{ 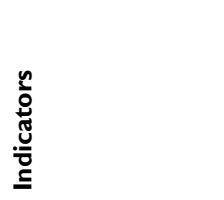 } & 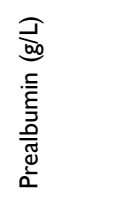 & 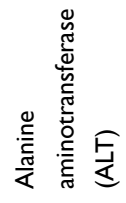 & 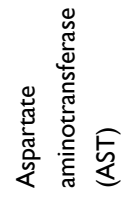 & tu & $\begin{array}{l}\frac{5}{5} \\
\frac{0}{0} \\
\frac{0}{U}\end{array}$ & 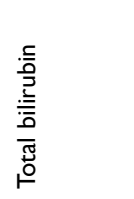 & 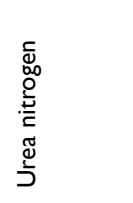 & $\begin{array}{l}\frac{0}{\bar{y}} \\
\stackrel{\breve{s}}{J}\end{array}$ \\
\hline
\end{tabular}




\begin{tabular}{|c|c|c|}
\hline $\begin{array}{l}\text { no } \\
\text { ô. }\end{array}$ & ֻัণ & $\overline{\bar{m}}$ \\
\hline 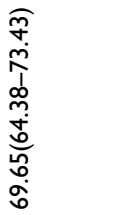 & 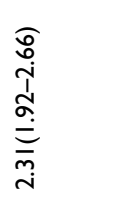 & $\begin{array}{l}\sigma \\
\frac{\sigma}{1} \\
\alpha \\
\sigma \\
\dot{0} \\
\frac{y}{i}\end{array}$ \\
\hline 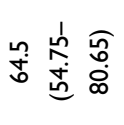 & 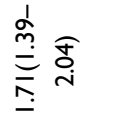 & $\begin{array}{l}\frac{1}{d} \\
\stackrel{0}{0} \\
\stackrel{0}{0} \\
\stackrel{0}{0} \\
0\end{array}$ \\
\hline 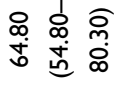 & 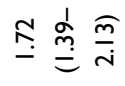 & 웅 $\stackrel{+}{\stackrel{0}{\infty}} \stackrel{0}{=}$ \\
\hline $\begin{array}{l}\stackrel{\infty}{\leftrightarrow} \\
\text { on } \\
o\end{array}$ & 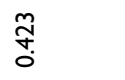 & 둥 \\
\hline 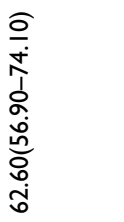 & 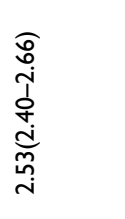 & 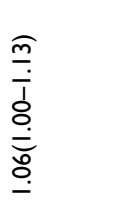 \\
\hline 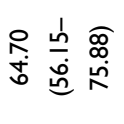 & 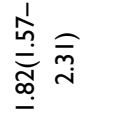 & $\begin{array}{l}\frac{1}{d} \\
\stackrel{\infty}{0} \\
\stackrel{0}{0} \\
\stackrel{\circ}{\circ} \\
\dot{0}\end{array}$ \\
\hline 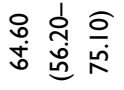 & 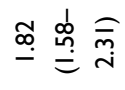 & 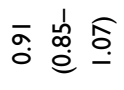 \\
\hline $\begin{array}{l}\text { L̊ } \\
\infty \\
0 \\
0\end{array}$ & $\frac{\text { 응 }}{0}$ & 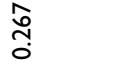 \\
\hline 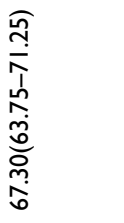 & 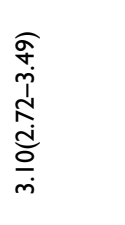 & 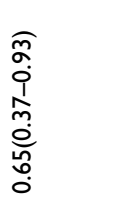 \\
\hline 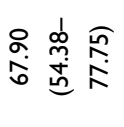 & 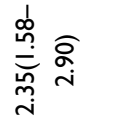 & 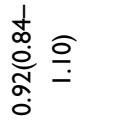 \\
\hline 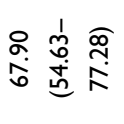 & 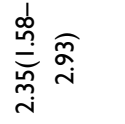 & 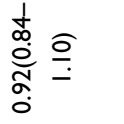 \\
\hline 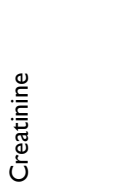 & 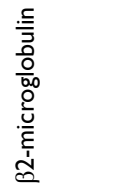 & $\begin{array}{l}\text { U } \\
\text { 㻤 } \\
\text { Uે }\end{array}$ \\
\hline
\end{tabular}

\section{Association of Serum Albumin with T Lymphocytes}

In our hypoalbuminemia test group, total numbers of $\mathrm{T}$ cells as well as $\mathrm{CD}^{+}$and $\mathrm{CD} 8^{+}$positive $\mathrm{T}$ cells were lower in patients with hypoalbuminemia compared to normal albumin controls (Table 4). Correlation analysis demonstrated that serum albumin was positively associated with the numbers for all 3 of these cell types (Figure 4). However, data points for weeks 1 and 3-4 were absent so conclusions of a correlation with patient recovery could not be made. Our results indicated that serum albumin levels in COVID-19 patients might be used as a biomarker to evaluate cellular immune functions.

\section{Association of Serum Albumin with Coagulation Function}

We also assessed coagulation functions in our patient groups and D dimer levels were significantly elevated in patients with hypoalbuminemia compared with patients with normal albumin. In contrast, PT, APTT and Fib levels were not significantly different from normal albumin controls (Table 5). These results revealed that serum albumin might be used as a predictor of coagulation function for COVID-19 patients.

\section{Discussion}

In the present study, we found that hypoalbuminemia was common in COVID-19 patients with severe disease. Moreover, the serum albumin levels gradually increased after treatment both in patients with normal serum albumin and hypoalbuminemia. We also found that serum albumin levels were positively correlated with lymphocyte and $\mathrm{RBC}$ numbers, $\mathrm{Hb}$ levels and total numbers of $\mathrm{T}$ cells and $\mathrm{CD}^{+}$and $\mathrm{CD} 8^{+}$cells and negatively correlated with CRP and AST. These results indicated that serum albumin concentrations might be used as a predicator of COVID-19 disease severity.

The half-life of serum albumin is 21 days $^{13}$ and a previous study indicated that when considering the period from onset to admission, hypoalbuminemia was less likely to be a result of decreased albumin synthesis in severe COVID-19 cases. $^{11}$ Although this was a reasonable assumption it has been challenged and the latter concluded that essential amino acid consumption due to viral replication, transcriptional inhibition and 


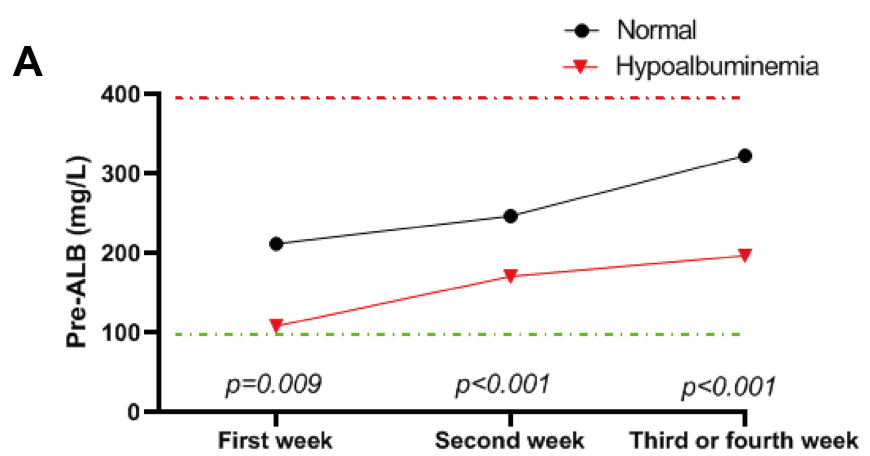

C

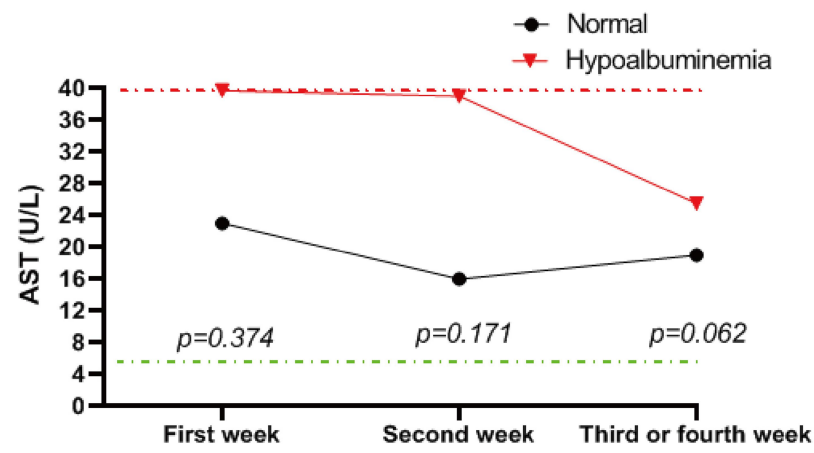

E

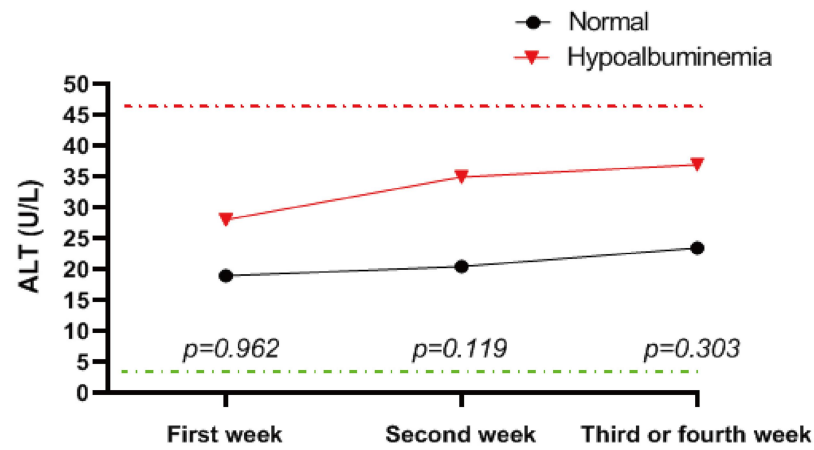

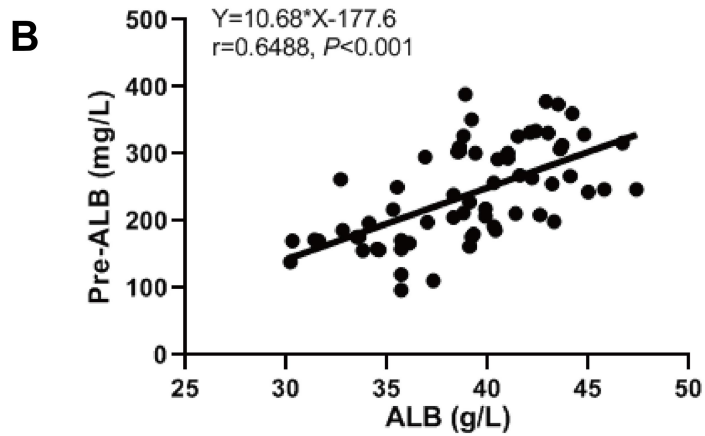

D

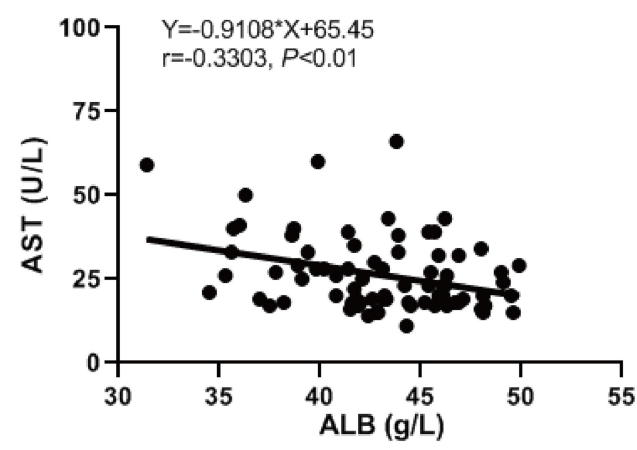

$\mathbf{F}$

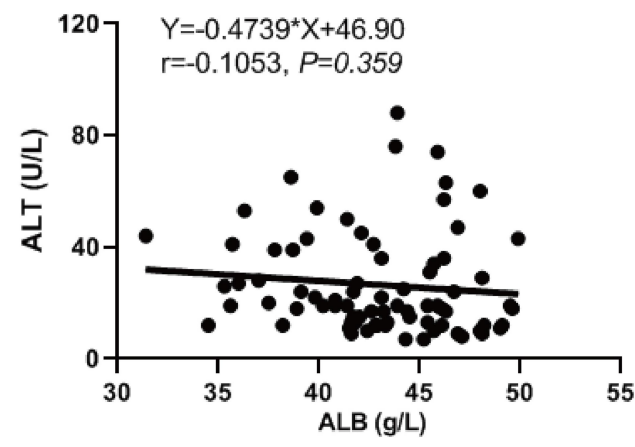

Figure 3 Dynamic analysis of prealbumin, ALT, and AST for COVID-19 patients. (A, C, E) Levels of prealbumin (A), ALT (C), andAST (E) in COVID-19 patients with albumin reduction and normal albumin at the indicated three time points.(B, D, F) The correlation of serum albumin with prealbumin (B), ALT (D), and AST (F) in COVID19 patients. Statistically significant at $P$ value less than 0.05 .

albumin clearance might be responsible for hypoalbuminemia in COVID-19 patients. $^{14}$ In our cohort, renal functional biomarkers including creatinine, $\beta 2$-microglobulin and cystatin $\mathrm{C}$ were not evidently different between patients with normal albumin and hypoalbuminemia. This indicated that albumin loss through the kidneys was not a likely reason for hypoalbuminemia in COVID-19 patients. In addition, ALT and AST were not elevated in patients with hypoalbuminemia but prealbumin levels were significantly decreased in the latter group. This indicated that SARSCoV-2 infection impaired liver protein synthesis functions and might be one of the causes of hypoalbuminemia in COVID-19 patients. We also found a negative 
Table 4 T Cell Counts in COVID-19 Patients with Normal Albumin or Hypoalbuminemia

\begin{tabular}{|l|c|c|c|c|}
\hline \multirow{2}{*}{ Indicators } & Total & \multicolumn{2}{|c|}{ Albumin } & \multirow{2}{*}{ P value } \\
\cline { 2 - 5 } & $\mathbf{n = 7 9}$ & Normal $(\mathbf{n = 6 5})$ & Hypoalbuminemia (n= 14) & \\
\hline Total T cells & $737.00(481.25-1061.00)$ & $970.50(589.00-1122.00)$ & $271.00(186.00-511.00)$ & 0.004 \\
CD4+ T cells & $416.00(241.50-658.50)$ & $522.00(351.00-676.00)$ & $147.00(109.00-296.00)$ & 0.007 \\
CD8+ T cells & $276.50(154.75-350.75)$ & $289.00(213.00-448.00)$ & $103.00(76.5 .00-202.50)$ & 0.004 \\
\hline
\end{tabular}

correlation between albumin and CRP suggesting that hypoalbuminemia may be associated with the increased microvascular permeability and consequent redistribution of albumin into extravascular compartments. This would result in a decrease in albumin in the blood vessels. Whether other factors contributed to the decrease of albumin in COVID-19 patients are unknown from these limited data and further studies are needed to elucidate the exact mechanisms.

The physiological functions of serum albumin include maintenance of osmotic pressure and transport of a variety of substances. ${ }^{13}$ After the outbreak of COVID-19, serum albumin was found significantly reduced in patients suffering from SARS-CoV-2. A recent study demonstrated that albumin binds to SARS-CoV-2 virions and this process might inhibit the formation of the endothelial glycocalyx by inhibition of albumin transport-binding sites. These authors suggested that albumin therapy should be tested as a matter of urgency in patients presenting with disseminated COVID-19 disease. ${ }^{15}$ In contrast, other reports indicated a cautious approach to albumin transfusion for COVID19 treatment before the exact mechanism of hypoalbuminemia was clarified. A summary study of 9 publications attempted to clarify the underlying role of serum albumin in patients with COVID-19 infections. They concluded that albumin transfusion treatment for COVID-19 patients might have a limited benefit through the existing evidence based on ARDS and sepsis. A recent preliminary report demonstrated that albumin supplementation dampens hypercoagulability in COVID19 patients and prevented ischemic events. ${ }^{16}$ Overall, whether albumin supplementation to COVID-19 patients is beneficial is currently unknown.

In addition to the therapeutic role of albumin, a number of studies have used albumin levels as a prognostic tool in patients with SARS-CoV-2 infections. For instance, serum albumin levels $<35 \mathrm{~g} /$ $\mathrm{L}$ were found in $74 \%$ of the COVID-19 patients and this prevalence was higher for ICU cases and those that suffered ischemic events. ${ }^{17}$ In our cohort, we found that D dimer levels were significantly elevated in patients with hypoalbuminemia, while no ischemic events were observed. Taken together, we collected data from three time points including within the first, second and third/fourth weeks. The dynamic analysis of serum albumin indicated that albumin levels were elevated after patients were recovering and indicated that serum albumin might be used as
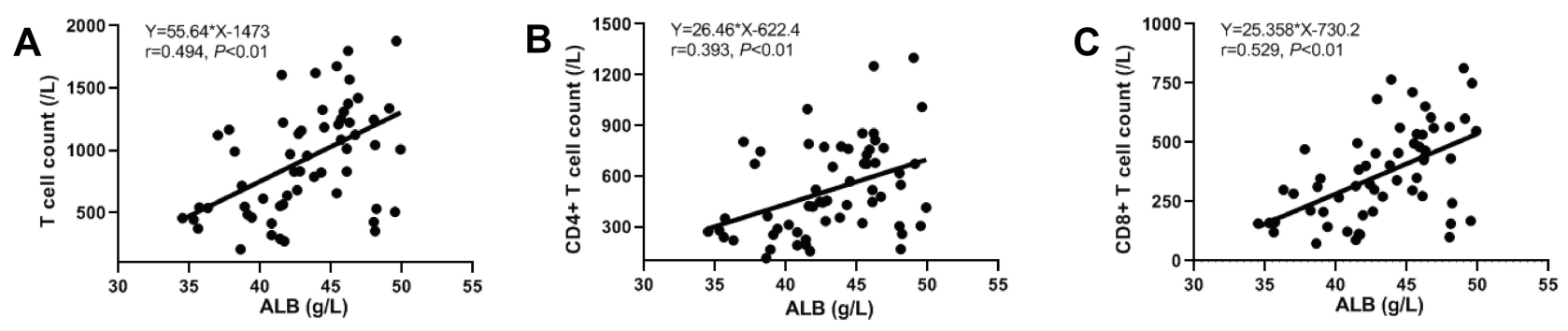

Figure 4 Correlation of serum albumin with total T lymphocytes count, CD4+ T lymphocytes, and CD8+ T lymphocytes. (A) Correlation of serum albumin with total T lymphocytes count. (B) Correlation of serum albumin with CD4+ T lymphocytes. (C) Correlation of serum albumin with CD8+ T lymphocytes. Statistically significant at $P$ value less than 0.05 . 
Table 5 The Coagulation Function of COVID-19 Patients with Normal Albumin or Hypoalbuminemia

\begin{tabular}{|l|c|c|c|c|}
\hline \multirow{2}{*}{ Indicators } & \multicolumn{3}{|c|}{ Albumin } & \multirow{2}{*}{ P value } \\
\cline { 2 - 5 } & Total $\mathbf{n = 7 9}$ & Normal $(\mathbf{n = 6}$ I) & Hypoalbuminemia (n=18) & \\
\hline PT & $11.60(10.88-12.13)$ & $11.70(10.50-12.20)$ & $11.40(11.20-11.95)$ & 0.899 \\
APTT & $35.35(28.65-38.00)$ & $35.30(28.80-37.10)$ & $35.50(28.15-41.15)$ & 0.824 \\
FIB & $3.69(2.92-4.71)$ & $3.24(2.68-4.37)$ & $4.29(4.08-4.86)$ & 0.053 \\
DD & $0.23(0.12-1.00)$ & $0.20(0.09-0.28)$ & $2.37(1.92-5.37)$ & 0.003 \\
\hline
\end{tabular}

a biomarker to predict the progress of COVID-19 disease.

\section{Data Sharing Statement}

The data used and/or analyzed during the current study are available from the corresponding author on reasonable request.

\section{Ethics Approval and Informed Consent}

The study protocol has been approved by the Medical Ethical Committee of the Chongqing General Hospital, University of Chinese Academy of Sciences with an approval number [S2020-021-01] and performed according to the Declaration of Helsinki. Due to the nature of this retrospective study without any additional interventions, patients' informed consents were exempted. All personally identifiable records were kept confidential.

\section{Disclosure}

The authors declare that they have no competing interests.

\section{References}

1. Arentz M, Yim E, Klaff L, et al. characteristics and outcomes of 21 critically ill patients with Covid-19 in Washington State. JAMA. 2020;323(16):1612-1614. doi:10.1001/jama.2020.4326

2. Matthay MA, Aldrich JM, Gotts JE. Treatment for severe acute respiratory distress syndrome from Covid-19. Lancet Respir Med. 2020;8(5):433-434. doi:10.1016/S2213-2600(20)30127-2

3. Roychoudhury S, Das A, Jha NK, et al. Viral pathogenesis of SARS-CoV-2 infection and male reproductive health. Open Biol. 2021;11(1):200347. doi:10.1098/rsob.200347

4. Mayet V, Mousseaux C, Petit-Hoang C, et al. Covid-19-associated acute kidney injury: after the tubule and the glomerulus, now the vessel? Clin Kidney J. 2020;13(6):1105-1106. doi:10.1093/ckj/sfaa210
5. Iqbal QZ, Haider MA, Sattar SBA, Hanif M, Javid I. Covid-19 induced myocarditis: a rare cause of heart failure. Cureus. 2020;12 (11):e11690. doi:10.7759/cureus.11690

6. Yao Y, Yuan X, Wu L, Guo N, Yin L, Li Y. Covid-19 and male reproduction: current research and unknown factors. Andrology. 2021. doi:10.1111/andr.12970

7. Mameli S, Marcialis MA, Bassareo PP, Fanos V. Covid-19 and hepatic damage: what we know? A systematic review. Panminerva Med. 2021. doi:10.23736/S0031-0808.21.04239-7

8. Huang W, Li C, Wang Z, et al. Decreased serum albumin level indicates poor prognosis of Covid-19 patients: hepatic injury analysis from 2623 hospitalized cases. Sci China Life Sci. 2020;63 (11):1678-1687. doi:10.1007/s11427-020-1733-4

9. Guan WJ, Liang WH, Zhao Y, et al. Comorbidity and its impact on 1590 patients with Covid-19 in China: a nationwide analysis. Eur Respir J. 2020;55(5):2000547. doi:10.1183/13993003.00547-2020

10. Guan WJ, Ni ZY, Hu Y, et al. Clinical characteristics of coronavirus disease 2019 in China. N Engl J Med. 2020;382(18):1708-1720. doi:10.1056/NEJMoa2002032

11. Huang J, Cheng A, Kumar R, et al. Hypoalbuminemia predicts the outcome of Covid-19 independent of age and co-morbidity. $J$ Med Virol. 2020;92(10):2152-2158. doi:10.1002/jmv.26003

12. de la Rica R, Borges M, Aranda M, et al. Low albumin levels are associated with poorer outcomes in a case series of Covid-19 patients in Spain: a retrospective cohort study. Microorganisms. 2020;8 (8):1106. doi:10.3390/microorganisms8081106

13. Rothschild MA, Oratz M, Schreiber SS. Serum albumin. Hepatology. 1988;8(2):385-401. doi:10.1002/hep.1840080234

14. Ambade V. Biochemical rationale for hypoalbuminemia in Covid-19 patients. J Med Virol. 2021;93(3):1207-1209. doi:10.1002/jmv.26542

15. Johnson AS, Fatemi R, Winlow W. SARS-CoV-2 Bound Human Serum Albumin and Systemic Septic Shock. Front Cardiovasc Med. 2020;7:153. doi:10.3389/fcvm.2020.00153

16. Violi F, Ceccarelli G, Loffredo L, et al. Albumin supplementation dampens hypercoagulability in Covid-19: a preliminary report. Thromb Haemost. 2021;121(1):102-105. doi:10.1055/s-00401721486

17. Violi F, Ceccarelli G, Cangemi R, et al. Hypoalbuminemia, coagulopathy, and vascular disease in Covid-19. Circ Res. 2020;127 (3):400-401. doi:10.1161/CIRCRESAHA.120.317173 


\section{Publish your work in this journal}

The International Journal of General Medicine is an international, peer-reviewed open-access journal that focuses on general and internal medicine, pathogenesis, epidemiology, diagnosis, monitoring and treatment protocols. The journal is characterized by the rapid reporting of reviews, original research and clinical studies across all disease areas. The manuscript management system is completely online and includes a very quick and fair peer-review system, which is all easy to use. Visit http://www.dovepress.com/ testimonials.php to read real quotes from published authors.

Submit your manuscript here: https://www.dovepress.com/international-journal-of-general-medicine-journal 